\title{
14.
}

\section{Theorie der Dreh- und Flieh-momente der paral- lelen Seitenkräfte, in welche Kräfte im Raume zerlegt werden können.}

(Vom Herrn geh. Rathe und Professor Dr. Schweins in Heidelberg.)

S. 1.

Bisher kannte man nur die Gleichungen zwischen den Drehmomenten um verschiedene Axen, aber nicht die Gleichungen zwischen ihren Bestandtheilen oder zwischen den Drehmomenten der parallelen Seitenkräfte, welche durch Zerlegen der Kräfte nach den Richtungen der Coordinatenaxen entstehen. Diese Gleichungen sollen hier entwickelt werden. Ihre Kenntnifs ist nützlich, was sich bei späteren Untersuchungen bewähren wird.

Die Bezeichnung ist folgende: ich nehme zwei rechtwinklichte Coordinatensysteme an $x, y, z$ und $x^{\prime}, y^{\prime}, z^{\prime}$, und nenne die Cosinusse der Winkel, welche

$$
\begin{aligned}
& x^{\prime} \text { mit } x, y, z \text { bildet, } \alpha, \beta, \gamma \\
& y^{\prime} \text {. . . . . . } \alpha^{\prime}, \beta^{\prime}, \gamma^{\prime} \\
& \Xi^{\prime} \cdot . \quad . \quad . \quad . \quad \alpha^{\prime \prime}, \beta^{\prime \prime}, \gamma^{\prime \prime}
\end{aligned}
$$

und die Coordinaten des Anfangspunctes des zweiten Coordinatensystems $\boldsymbol{a}, \boldsymbol{b}, \boldsymbol{c}$. Ferner selze ich

$$
\begin{aligned}
& \Sigma \boldsymbol{X}=\boldsymbol{A}, \quad \Sigma \mathrm{y} \boldsymbol{Z}=\boldsymbol{F}, \quad \Sigma \mathrm{z} \boldsymbol{Y}=\boldsymbol{F}_{1}, \quad \Sigma x \boldsymbol{X}=\boldsymbol{l}, \quad l+m=h \\
& \Sigma \boldsymbol{Y}=\boldsymbol{B}, \quad \Sigma_{\boldsymbol{z}} \boldsymbol{X}=\boldsymbol{G}, \quad \Sigma x \boldsymbol{Z}=\boldsymbol{G}_{1}, \quad \Sigma y \boldsymbol{Y}=m, \quad l+n=y \\
& \Sigma \mathbb{Z}=\boldsymbol{C}, \quad \Sigma x \boldsymbol{Y}=\boldsymbol{H}, \quad \Sigma y X=H_{1}, \quad \Sigma z \boldsymbol{Z}=n, \quad m+n=f
\end{aligned}
$$

und

$$
\begin{aligned}
& \Sigma \boldsymbol{X}^{\prime}=\boldsymbol{A}^{\prime}, \quad \Sigma y^{\prime} \mathbf{Z}^{\prime}=\boldsymbol{H}^{\prime \prime}, \quad \Sigma \boldsymbol{z}^{\prime} \boldsymbol{Y}^{\prime}=\boldsymbol{H}^{\prime \prime}, \quad \Sigma \boldsymbol{x}^{\prime} \boldsymbol{X}^{\prime}=\boldsymbol{l}^{\prime}, \quad \boldsymbol{l}^{\prime}+\boldsymbol{m}^{\prime}=\boldsymbol{l}^{\prime} \\
& \Sigma \boldsymbol{Y}^{\prime}=\boldsymbol{B}^{\prime}, \quad \Sigma \boldsymbol{z}^{\prime} \boldsymbol{X}^{\prime}=\boldsymbol{G}^{\prime}, \quad \Sigma \boldsymbol{x}^{\prime} \mathbf{Z}^{\prime}=\boldsymbol{G}^{\prime}, \quad \Sigma \boldsymbol{y}^{\prime} \boldsymbol{Y}^{\prime}=\boldsymbol{m}^{\prime}, \quad \boldsymbol{l}^{\prime}+\boldsymbol{n}^{\prime}=\boldsymbol{g}^{\prime} \\
& \Sigma \mathbb{Z}^{\prime}=C^{\prime}, \quad \Sigma x^{\prime} \boldsymbol{Y}^{\prime}=H^{\prime}, \quad \Sigma y^{\prime} \boldsymbol{X}^{\prime}=H^{\prime}, \quad \Sigma z^{\prime} \mathbf{Z}^{\prime}=n^{\prime}, \quad m^{\prime}+n^{\prime}=f^{\prime}
\end{aligned}
$$

und

$$
\begin{aligned}
& \Sigma\left(y^{\prime} \boldsymbol{Z}-\boldsymbol{z} \boldsymbol{Y}\right)=\boldsymbol{H}-\boldsymbol{F}_{1}=\boldsymbol{L}, \quad \Sigma\left(y^{\prime} \boldsymbol{Z}^{\prime}-\boldsymbol{z}^{\prime} \boldsymbol{Y}^{\prime}\right)=\boldsymbol{F}^{\prime}-\boldsymbol{F}_{1}^{\prime}=\boldsymbol{L}^{\prime} \\
& \Sigma(\boldsymbol{z} \boldsymbol{X}-\boldsymbol{x} \mathbf{Z})=\boldsymbol{G}-\boldsymbol{G}_{1}=\boldsymbol{M}, \quad \Sigma\left(\boldsymbol{z}^{\prime} \boldsymbol{X}^{\prime}-\boldsymbol{x}^{\prime} \boldsymbol{Z}^{\prime}\right)=\boldsymbol{G}^{\prime}-\boldsymbol{G}_{1}^{\prime}=M^{\prime} \\
& \Sigma(x \boldsymbol{Y}-\boldsymbol{y} \boldsymbol{X})=\boldsymbol{H}-\boldsymbol{H}_{1}=\mathbf{N}, \quad \Sigma\left(x^{\prime} \boldsymbol{Y}^{\prime}-y^{\prime} \boldsymbol{X}^{\prime}\right)=\boldsymbol{H}^{\prime}-\boldsymbol{H}_{1}^{\prime}=\mathbf{N}^{\prime}
\end{aligned}
$$


und

$$
\begin{aligned}
& \Sigma((y-b) \boldsymbol{Z}-(\boldsymbol{z}-\boldsymbol{c}) \boldsymbol{Y})=\boldsymbol{L}_{b c} \\
& \Sigma((\boldsymbol{z}-\boldsymbol{c}) \boldsymbol{X}-(\boldsymbol{x}-\boldsymbol{a}) \boldsymbol{Z})=\boldsymbol{M}_{a c} \\
& \Sigma((x-\boldsymbol{a}) \boldsymbol{Y}-(\boldsymbol{y}-\boldsymbol{b}) \boldsymbol{X})=\boldsymbol{N}_{a b}
\end{aligned}
$$

und zuletzt

$$
\begin{aligned}
& \Sigma(y-b) \boldsymbol{Z}=\boldsymbol{F}_{b}, \quad \Sigma(\Sigma-c) \boldsymbol{Y}=\boldsymbol{H}_{1 c}, \quad \Sigma(x-a) \boldsymbol{X}=\boldsymbol{l}_{a}, \quad \boldsymbol{l}_{a}+m_{b}=\boldsymbol{h}_{a b} \\
& \Sigma(\boldsymbol{z}-\boldsymbol{c}) \boldsymbol{X}=\boldsymbol{G}_{c}, \quad \boldsymbol{\Sigma}(\boldsymbol{x}-\boldsymbol{a}) \boldsymbol{Z}=\boldsymbol{G}_{1 a}, \quad \boldsymbol{\Sigma}(\boldsymbol{y}-\boldsymbol{b}) \boldsymbol{Y}=\boldsymbol{m}_{b}, \boldsymbol{l}_{a}+\boldsymbol{n}_{c}=\boldsymbol{g}_{a c} \\
& \Sigma(x-a) \boldsymbol{Y}=\boldsymbol{H}_{a}, \quad \Sigma(y-b) X=H_{1 b}, \quad \Sigma(z-c) \boldsymbol{Z}=n_{c}, \quad m_{b}+n_{c}=f_{b c} \\
& \boldsymbol{u}_{a b c}=\boldsymbol{l}_{a}+\boldsymbol{m}_{b}+\boldsymbol{n}_{c}, \quad \boldsymbol{u}=\boldsymbol{l}+\boldsymbol{m}+\boldsymbol{n} \text {. }
\end{aligned}
$$

S. 2 .

Das Product

$$
(\boldsymbol{x}-\boldsymbol{a}) \cdot \mathbf{Z}^{\prime}
$$

läfst sich auf zwei verschiedene Weisen durch andere Producte ersetzen, nämlich, wenn entweder statt $\mathbf{Z}^{\prime}$

$$
\boldsymbol{Z}^{\prime}=\alpha^{\prime \prime} \boldsymbol{X}+\boldsymbol{\beta}^{\prime \prime} \boldsymbol{Y}+\gamma^{\prime \prime} \boldsymbol{Z}
$$

oder statt des anderen Factors $x-a$

$$
x-a=\alpha x^{\prime}+\alpha^{\prime} y^{\prime}+\alpha^{\prime \prime} z^{\prime}
$$

gesetzt wird. Man erhält dadurch die Gleichung

$$
(x-a)\left(\alpha^{\prime \prime} \boldsymbol{X}+\beta^{\prime \prime} \boldsymbol{Y}+\gamma^{\prime \prime} \boldsymbol{Z}\right)=\left(\alpha x^{\prime}+\alpha^{\prime} y^{\prime}+\alpha^{\prime \prime} \boldsymbol{z}^{\prime}\right) \mathbf{Z}^{\prime} .
$$

Eben so kann man bei den Producten

$$
(\boldsymbol{y}-\boldsymbol{b}) \mathbf{Z}^{\prime} \text { und }(\boldsymbol{z}-\boldsymbol{c}) \mathbf{Z}^{\prime}
$$

verfahren, nämlich

$$
\begin{aligned}
& (\boldsymbol{y}-\boldsymbol{b})\left(\alpha^{\prime \prime} \boldsymbol{X}+\boldsymbol{\beta}^{\prime \prime} \boldsymbol{Y}+\gamma^{\prime \prime} \boldsymbol{Z}\right)=\left(\boldsymbol{\beta} \boldsymbol{x}^{\prime}+\boldsymbol{\beta}^{\prime} \boldsymbol{y}^{\prime}+\boldsymbol{\beta}^{\prime \prime} \boldsymbol{z}^{\prime}\right) \boldsymbol{Z}^{\prime} \\
& (\boldsymbol{z}-\boldsymbol{c})\left(\alpha^{\prime \prime} \boldsymbol{X}+\boldsymbol{\beta}^{\prime \prime} \boldsymbol{Y}+\boldsymbol{\gamma}^{\prime \prime} \boldsymbol{Z}\right)=\left(\boldsymbol{\gamma} \boldsymbol{x}^{\prime}+\boldsymbol{\gamma}^{\prime} \boldsymbol{y}^{\prime}+\boldsymbol{\gamma}^{\prime \prime} \boldsymbol{z}^{\prime}\right) \boldsymbol{Z}^{\prime} .
\end{aligned}
$$

Wenn man nun den einzelnen Producten das Summenzeichen vorsetzt, und sich der obigen Zeichen bedient, so erhält man folgende Gleichungen:

$$
\left\{\begin{array}{l}
\alpha^{\prime \prime} \boldsymbol{l}_{a}+\beta^{\prime \prime} \boldsymbol{H}_{a}+\gamma^{\prime \prime} \boldsymbol{G}_{1 a}=\alpha \boldsymbol{G}_{1}^{\prime}+\alpha^{\prime} \boldsymbol{F}^{\prime \prime}+\alpha^{\prime \prime} n^{\prime} \\
\alpha^{\prime \prime} \boldsymbol{H}_{1 b}+\beta^{\prime \prime} \boldsymbol{m}_{b}+\gamma^{\prime \prime} \boldsymbol{F}_{b}=\beta \boldsymbol{G}_{1}^{\prime}+\boldsymbol{\beta}^{\prime} \boldsymbol{F}^{\prime \prime}+\beta^{\prime \prime} n^{\prime} \\
\alpha^{\prime \prime} \boldsymbol{G}_{c}+\beta^{\prime \prime} \boldsymbol{F}_{1 c}+\gamma^{\prime \prime} \boldsymbol{n}_{c}=\gamma \boldsymbol{G}_{1}^{\prime}+\gamma^{\prime} \boldsymbol{F}^{\prime \prime}+\gamma^{\prime \prime} \boldsymbol{n}^{\prime} .
\end{array}\right.
$$

Eben so behandele ich die Producte

$$
\boldsymbol{z}^{\prime} . \boldsymbol{X}, \quad \boldsymbol{z}^{\prime} . \boldsymbol{Y}, \quad \boldsymbol{z}^{\prime} . \mathbf{Z},
$$

ich ersetze den einen Factor durch andere Gröfsen, während der zweite 
Factor unverändert bleibt, nämlich

$$
\begin{aligned}
& \left(\alpha^{\prime \prime}(x-a)+\beta^{\prime \prime}(y-b)+\gamma^{\prime \prime}(z-c)\right) \boldsymbol{X}=\boldsymbol{z}^{\prime}\left(\alpha \boldsymbol{X}^{\prime}+\alpha^{\prime} \boldsymbol{Y}^{\prime}+\alpha^{\prime \prime} \boldsymbol{Z}^{\prime}\right) \\
& \text { ( . . . . . . . . . . . ) } \boldsymbol{Y}=\boldsymbol{z}^{\prime}\left(\boldsymbol{\beta} \boldsymbol{X}^{\prime}+\beta^{\prime} \boldsymbol{Y}^{\prime}+\beta^{\prime \prime} \boldsymbol{Z}^{\prime}\right) \\
& \text { (. . . . . . . . . . }) \boldsymbol{Z}=\boldsymbol{z}^{\prime}\left(\boldsymbol{\gamma} \boldsymbol{X}^{\prime}+\gamma^{\prime} \boldsymbol{Y}^{\prime}+\gamma^{\prime \prime} \boldsymbol{Z}^{\prime}\right) \text {, }
\end{aligned}
$$

und erhalte folgende Gleichungen:

$$
\left\{\begin{array}{l}
\alpha^{\prime \prime} l_{a}+\beta^{\prime \prime} \boldsymbol{H}_{1 b}+\gamma^{\prime \prime} \boldsymbol{G}_{c}=\alpha \boldsymbol{G}^{\prime}+\alpha^{\prime} \boldsymbol{F}_{1}^{\prime \prime}+\alpha^{\prime \prime} \boldsymbol{n}^{\prime} \\
\alpha^{\prime \prime} \boldsymbol{H}_{a}+\beta^{\prime \prime} \boldsymbol{m}_{b}+\gamma^{\prime \prime} \boldsymbol{F}_{\mathbf{1}}=\beta \boldsymbol{G}^{\prime}+\boldsymbol{\beta}^{\prime} \boldsymbol{F}_{\mathbf{1}}^{\prime}+\boldsymbol{\beta}^{\prime \prime} \boldsymbol{n}^{\prime} \\
\alpha^{\prime \prime} \boldsymbol{G}_{\mathbf{1}}+\boldsymbol{\beta}^{\prime \prime} \boldsymbol{F}_{b}+\gamma^{\prime \prime} \boldsymbol{n}_{c}=\gamma \boldsymbol{G}^{\prime}+\gamma^{\prime} \boldsymbol{F}_{\mathbf{1}}^{\prime}+\gamma^{\prime \prime} \boldsymbol{n}^{\prime} .
\end{array}\right.
$$

Werden die Producte

$$
(x-a) \boldsymbol{Y}^{\prime}, \quad(y-b) \boldsymbol{Y}^{\prime}, \quad(z-c) \boldsymbol{Y}^{\prime}, \quad y^{\prime} \boldsymbol{X}, \quad y^{\prime} \boldsymbol{Y}, \quad y^{\prime} \boldsymbol{Z}
$$

einer gleichen Behandlung unterworfen, so entstehen die Gleichungen

$$
\left\{\begin{array}{l}
\alpha^{\prime} \boldsymbol{l}_{a}+\beta^{\prime} \boldsymbol{H}_{a}+\gamma^{\prime} \boldsymbol{G}_{1 a}=\alpha \boldsymbol{H}^{\prime}+\alpha^{\prime} \boldsymbol{m}^{\prime}+\alpha^{\prime \prime} \boldsymbol{H}_{1}^{\prime \prime} \\
\alpha^{\prime} \boldsymbol{H}_{1 b}+\beta^{\prime} \boldsymbol{m}_{b}+\gamma^{\prime} \boldsymbol{F}_{b}=\beta \boldsymbol{H}^{\prime}+\beta^{\prime} \boldsymbol{m}^{\prime}+\beta^{\prime \prime} \boldsymbol{F}_{1}^{\prime \prime} \\
\alpha^{\prime} \boldsymbol{G}_{c}+\boldsymbol{\beta}^{\prime} \boldsymbol{F}_{1 c}+\gamma^{\prime} \boldsymbol{n}_{c}=\gamma \boldsymbol{H}^{\prime}+\gamma^{\prime} \boldsymbol{m}^{\prime}+\gamma^{\prime \prime} \boldsymbol{F}_{1}^{\prime \prime}
\end{array}\right.
$$

und

$$
\left\{\begin{array}{l}
\alpha^{\prime} \boldsymbol{l}_{a}+\boldsymbol{\beta}^{\prime} \boldsymbol{H}_{1 b}+\gamma^{\prime} \boldsymbol{G}_{c}=\alpha \boldsymbol{H}_{\mathbf{1}}^{\prime}+\alpha^{\prime} \boldsymbol{m}^{\prime}+\alpha^{\prime \prime} \boldsymbol{F}^{\prime \prime} \\
\alpha^{\prime} \boldsymbol{H}_{a}+\boldsymbol{\beta}^{\prime} \boldsymbol{m}_{b}+\gamma^{\prime} \boldsymbol{F}_{\mathbf{1}}=\boldsymbol{\beta} \boldsymbol{H}_{\mathbf{1}}^{\prime}+\boldsymbol{\beta}^{\prime} \boldsymbol{m}^{\prime}+\boldsymbol{\beta}^{\prime \prime} \boldsymbol{F}^{\prime \prime} \\
\alpha^{\prime} \boldsymbol{G}_{\mathbf{1}}+\boldsymbol{\beta}^{\prime} \boldsymbol{F}_{b}+\gamma^{\prime} \boldsymbol{n}_{c}=\gamma \boldsymbol{H}_{\mathbf{1}}^{\prime}+\gamma^{\prime} \boldsymbol{m}^{\prime}+\gamma^{\prime \prime} \boldsymbol{F}^{\prime \prime} .
\end{array}\right.
$$

Zuletzt erzeugen die Producte

$$
(x-a) \boldsymbol{X}^{\prime}, \quad(y-b) \boldsymbol{X}^{\prime}, \quad(z-c) \boldsymbol{X}^{\prime}, \quad x^{\prime} \boldsymbol{X}, \quad x^{\prime} \boldsymbol{Y}, \quad x^{\prime} \boldsymbol{Z}
$$

bei einem gleichen Verfahren die Gleichungen

$$
\left\{\begin{array}{l}
\alpha \boldsymbol{l}_{a}+\beta \boldsymbol{H}_{a}+\gamma \boldsymbol{G}_{1 a}=\alpha l^{\prime}+\alpha^{\prime} \boldsymbol{H}_{1}^{\prime}+\alpha^{\prime \prime} \boldsymbol{G}^{\prime} \\
\alpha \boldsymbol{H}_{1 b}+\beta m_{b}+\gamma \boldsymbol{F}_{b}=\beta \boldsymbol{l}^{\prime}+\beta^{\prime} \boldsymbol{H}_{1}^{\prime}+\beta^{\prime \prime} \boldsymbol{G}^{\prime} \\
\alpha \boldsymbol{G}_{c}+\beta \boldsymbol{H}_{1 c}+\gamma \boldsymbol{n}_{c}=\gamma \boldsymbol{l}^{\prime}+\gamma^{\prime} \boldsymbol{H}_{1}^{\prime}+\gamma^{\prime \prime} \boldsymbol{G}^{\prime}
\end{array}\right.
$$

und

$$
\left\{\begin{array}{l}
\alpha l_{a}+\beta \boldsymbol{H}_{1 b}+\gamma \boldsymbol{G}_{c}=\alpha l^{\prime}+\alpha^{\prime} \boldsymbol{H}^{\prime}+\alpha^{\prime \prime} \boldsymbol{G}_{1}^{\prime} \\
\alpha \boldsymbol{H}_{a}+\beta m_{b}+\gamma \boldsymbol{F}_{1 c}=\beta l^{\prime}+\beta^{\prime} \boldsymbol{H}^{\prime}+\beta^{\prime \prime} \boldsymbol{G}_{1}^{\prime} \\
\alpha \boldsymbol{G}_{1 a}+\beta \boldsymbol{F}_{b}+\gamma \boldsymbol{n}_{c}=\gamma l^{\prime}+\gamma^{\prime} \boldsymbol{H}^{\prime}+\beta^{\prime \prime} \boldsymbol{G}_{1}^{\prime} .
\end{array}\right.
$$

Diese sechs Gleichungen geben den Zusammenhang der Dreh- und der Fliehmomente der parallelen Seitenkräfte in einem rechtwinklichten Coordinatensysteme mit den Dreh- und Flieh-momenten der Seitenkräfte in anderen rechtwinklichten Coordinatensystemen an. 
S. 3.

Die Producte

$$
\boldsymbol{l}_{a}, \boldsymbol{m}_{b}, \boldsymbol{n}_{c}, \quad \boldsymbol{l}^{\prime}, \boldsymbol{m}^{\prime}, \boldsymbol{n}^{\prime},
$$

welche in diesen Gleichungen vorkommen, können durch andere ersetzt werden. Es ist nämlich nach meiner früheren Untersuchung über Fliehmomente im 38. Band 1. Heft dieses Journals Nr. 21.

$$
\boldsymbol{l}_{a}+\boldsymbol{m}_{b}+\boldsymbol{n}_{c}=\boldsymbol{l}^{\prime}+\boldsymbol{m}^{\prime}+\boldsymbol{n}^{\prime}=\boldsymbol{U}_{a b c}
$$

eine beständige Gröfse, denn die verschiedene Richtung der Coordinaten hat auf dieselbe keinen Einflufs; daher

$$
\begin{aligned}
\boldsymbol{l}_{a} & =\boldsymbol{u}_{a b c}-f_{b c}, & \boldsymbol{l}^{\prime} & =\boldsymbol{u}_{a b c}-f^{\prime} \\
\boldsymbol{m}_{b} & =\boldsymbol{u}_{a b c}-\boldsymbol{g}_{a c}, & \boldsymbol{m}^{\prime} & =\boldsymbol{u}_{a b c}-\boldsymbol{g}^{\prime} \\
\boldsymbol{n}_{c} & =\boldsymbol{u}_{a b c}-\boldsymbol{h}_{a b}, & \boldsymbol{n}^{\prime} & =\boldsymbol{u}_{a b c}-\boldsymbol{h}^{\prime} .
\end{aligned}
$$

Werden diese Producte statt der ersteren gesetzt, so fällt wieder die beständige Gröfse aus den Gleichungen, und diese gehen in folgende über:

und

$$
\left\{\begin{array}{l}
-\alpha^{\prime \prime} f_{b c}+\beta^{\prime \prime} \boldsymbol{H}_{a}+\gamma^{\prime \prime} \boldsymbol{G}_{1 a}=\alpha \boldsymbol{G}_{1}^{\prime}+\alpha^{\prime} \boldsymbol{F}^{\prime}-\alpha^{\prime \prime} \boldsymbol{h}^{\prime} \\
+\alpha^{\prime \prime} \boldsymbol{H}_{1 b}+\beta^{\prime \prime} \boldsymbol{g}_{a c}+\gamma^{\prime \prime} \boldsymbol{F}_{b}=\beta \boldsymbol{G}_{\mathbf{1}}^{\prime}+\beta^{\prime} \boldsymbol{F}^{\prime \prime}-\beta^{\prime \prime} \boldsymbol{h}^{\prime} \\
+\alpha^{\prime \prime} \boldsymbol{G}_{c}+\beta^{\prime \prime} \boldsymbol{F}_{1 c}-\gamma^{\prime \prime} \boldsymbol{h}_{a b}=\gamma \boldsymbol{G}_{\mathbf{1}}^{\prime}+\gamma^{\prime} \boldsymbol{F}^{\prime}-\gamma^{\prime \prime} \boldsymbol{h}^{\prime}
\end{array}\right.
$$

$$
\begin{aligned}
& \left\{\begin{array}{l}
-\alpha^{\prime \prime} f_{b c}+\beta^{\prime \prime} \boldsymbol{H}_{1 b}+\gamma^{\prime \prime} \boldsymbol{G}_{c}=\alpha \boldsymbol{G}^{\prime}+\alpha^{\prime} \boldsymbol{F}_{1}^{\prime}-\alpha^{\prime \prime} \boldsymbol{h}^{\prime} \\
+\alpha^{\prime \prime} \boldsymbol{H}_{a}-\beta^{\prime \prime} \boldsymbol{g}_{a c}+\gamma^{\prime \prime} \boldsymbol{F}_{1 c}=\beta \boldsymbol{G}^{\prime}+\boldsymbol{\beta}^{\prime} \boldsymbol{H}_{1}^{\prime}-\boldsymbol{\beta}^{\prime \prime} \boldsymbol{h}^{\prime} \\
+\alpha^{\prime \prime} \boldsymbol{G}_{1 a}+\beta^{\prime \prime} \boldsymbol{F}_{b}+\gamma^{\prime \prime} \boldsymbol{h}_{a b}=\gamma \boldsymbol{G}^{\prime}+\gamma^{\prime} \boldsymbol{F}_{1}^{\prime}-\gamma^{\prime \prime} \boldsymbol{h}^{\prime},
\end{array}\right. \\
& \left\{\begin{array}{l}
-\alpha^{\prime} f_{b c}+\beta^{\prime} \boldsymbol{H}_{a}+\gamma^{\prime} \boldsymbol{G}_{1 a}=\alpha \boldsymbol{H}^{\prime}-\alpha^{\prime} \boldsymbol{g}^{\prime}+\alpha^{\prime \prime} \boldsymbol{H}_{\mathbf{1}}^{\prime \prime} \\
+\alpha^{\prime} \boldsymbol{H}_{1 b}-\beta^{\prime} \boldsymbol{g}_{a c}+\gamma^{\prime} \boldsymbol{F}_{b}=\beta \boldsymbol{H}^{\prime}-\beta^{\prime} \boldsymbol{g}^{\prime}+\boldsymbol{\beta}^{\prime \prime} \boldsymbol{F}_{\mathbf{1}}^{\prime \prime} \\
+\alpha^{\prime} \boldsymbol{G}_{c}+\beta^{\prime} \boldsymbol{H}_{1 c}-\gamma^{\prime} \boldsymbol{h}_{a b}=\gamma \boldsymbol{H}^{\prime}-\gamma^{\prime} \boldsymbol{g}^{\prime}+\gamma^{\prime \prime} \boldsymbol{H}_{\mathbf{1}}^{\prime},
\end{array}\right. \\
& \left\{\begin{array}{l}
-\alpha^{\prime} f_{b c}+\beta^{\prime} \boldsymbol{H}_{1 b}+\gamma^{\prime} \boldsymbol{G}_{c}=\alpha \boldsymbol{H}_{\mathbf{1}}^{\prime}-\alpha^{\prime} \boldsymbol{g}^{\prime}+\alpha^{\prime \prime} \boldsymbol{H}^{\prime \prime} \\
+\alpha^{\prime} \boldsymbol{H}_{a}-\beta^{\prime} \boldsymbol{g}_{a c}+\gamma^{\prime} \boldsymbol{F}_{1 c}=\beta \boldsymbol{H}_{\mathbf{1}}^{\prime}-\beta \boldsymbol{g}^{\prime}+\beta^{\prime \prime} \boldsymbol{F}^{\prime \prime} \\
+\alpha^{\prime} \boldsymbol{G}_{\mathbf{1}}+\boldsymbol{\beta}^{\prime} \boldsymbol{F}_{b}-\gamma^{\prime} \boldsymbol{h}_{a b}=\gamma \boldsymbol{H}_{\mathbf{1}}^{\prime}-\gamma^{\prime} \boldsymbol{g}^{\prime}+\boldsymbol{\gamma}^{\prime \prime} \boldsymbol{F}^{\prime \prime},
\end{array}\right. \\
& \left\{\begin{array}{l}
-\alpha \boldsymbol{f}_{b c}+\beta \boldsymbol{H}_{a}+\gamma \boldsymbol{G}_{1 a}=-\alpha \boldsymbol{f}^{\prime}+\alpha^{\prime} \boldsymbol{H}_{\mathbf{1}}^{\prime}+\alpha^{\prime \prime} \boldsymbol{G}^{\prime} \\
+\alpha \boldsymbol{H}_{1 b}-\beta \boldsymbol{g}_{a c}+\gamma \boldsymbol{F}_{b}=-\boldsymbol{\beta} \boldsymbol{f}^{\prime}+\boldsymbol{\beta}^{\prime} \boldsymbol{H}_{\mathbf{1}}^{\prime}+\boldsymbol{\beta}^{\prime \prime} \boldsymbol{G}^{\prime} \\
+\alpha \boldsymbol{G}_{c}+\boldsymbol{\beta} \boldsymbol{F}_{1 c}-\boldsymbol{\gamma} \boldsymbol{h}_{a b}=-\gamma \boldsymbol{f}^{\prime}+\boldsymbol{\gamma}^{\prime} \boldsymbol{H}_{\mathbf{1}}^{\prime}+\boldsymbol{\gamma}^{\prime \prime} \boldsymbol{G}^{\prime},
\end{array}\right. \\
& \left\{\begin{array}{l}
-\alpha f_{b c}+\beta \boldsymbol{H}_{1 b}+\gamma \boldsymbol{G}_{c}=-\alpha \boldsymbol{f}^{\prime}+\alpha^{\prime} \boldsymbol{H}^{\prime}+\alpha^{\prime \prime} \boldsymbol{G}_{\mathbf{1}}^{\prime} \\
+\alpha \boldsymbol{H}_{a}-\beta \boldsymbol{g}_{a c}+\gamma \boldsymbol{H}_{1 c}=-\boldsymbol{\beta} \boldsymbol{f}^{\prime}+\boldsymbol{\beta}^{\prime} \boldsymbol{H}^{\prime}+\boldsymbol{\beta}^{\prime \prime} \boldsymbol{G}_{\mathbf{1}}^{\prime} \\
+\alpha \boldsymbol{G}_{\mathbf{1}}+\boldsymbol{\beta} \boldsymbol{F}_{b}-\gamma \boldsymbol{h}_{a b}=-\gamma \boldsymbol{f}^{\prime}+\boldsymbol{\gamma}^{\prime} \boldsymbol{H}^{\prime}+\boldsymbol{\gamma}^{\prime \prime} \boldsymbol{G}_{\mathbf{1}}^{\prime} .
\end{array}\right.
\end{aligned}
$$




\section{S. 4.}

Die Gleichungen, wodurch die Summen der Producte

$$
x^{\prime} \boldsymbol{X}^{\prime}, y^{\prime} \boldsymbol{Y}^{\prime}, z^{\prime} \boldsymbol{Z}^{\prime}, x^{\prime} \boldsymbol{Y}^{\prime}, x^{\prime} \boldsymbol{Z}^{\prime}, y^{\prime} \boldsymbol{X}^{\prime}, y^{\prime} \boldsymbol{Z}^{\prime}, \boldsymbol{z}^{\prime} \boldsymbol{X}^{\prime}, \boldsymbol{z}^{\prime} \boldsymbol{Y}^{\prime}
$$

in einem Coordinatensysteme durch die Summen im anderen Coordinatensysteme dargestellt werden, sind

(13.) $\Sigma \boldsymbol{x}^{\prime} \boldsymbol{X}^{\prime}=\boldsymbol{l}^{\prime}=\alpha^{2} \boldsymbol{l}_{a}+\beta^{2} \boldsymbol{m}_{b}+\gamma^{2} \boldsymbol{n}_{c}+\alpha \beta\left(\boldsymbol{H}_{a}+\boldsymbol{H}_{\mathbf{1}}\right)+\alpha \gamma\left(\boldsymbol{G}_{\mathbf{1}}+\boldsymbol{G}_{c}\right)+\beta \gamma\left(\boldsymbol{F}_{b}+\boldsymbol{F}_{\mathbf{1}}\right)$

$$
\begin{gathered}
=\alpha^{2} \boldsymbol{l}+\boldsymbol{\beta}^{2} \boldsymbol{m}+\boldsymbol{\gamma}^{2} \boldsymbol{n}+\alpha \boldsymbol{\beta}\left(\boldsymbol{H}+\boldsymbol{H}_{\mathbf{1}}\right)+\alpha \boldsymbol{\gamma}\left(\boldsymbol{G}_{\mathbf{1}}+\boldsymbol{G}\right)+\beta \gamma\left(\boldsymbol{F}+\boldsymbol{F}_{\mathbf{1}}\right) \\
-(\alpha \boldsymbol{A}+\beta \boldsymbol{B}+\boldsymbol{\gamma} \boldsymbol{C})(\boldsymbol{\alpha} \boldsymbol{a}+\boldsymbol{\beta} \boldsymbol{b}+\boldsymbol{\gamma} \boldsymbol{c}),
\end{gathered}
$$

(14.) $\Sigma y^{\prime} \boldsymbol{Y}^{\prime}=m^{\prime}=\alpha^{\prime 2} l_{a}+\beta^{\prime 2} m_{b}+\gamma^{\prime 2} n_{c}+\alpha^{\prime} \beta^{\prime}\left(\boldsymbol{H}_{a}+\boldsymbol{H}_{1 b}\right)+\alpha^{\prime} \gamma^{\prime}\left(\boldsymbol{G}_{1 a}+\boldsymbol{G}_{c}\right)+\beta^{\prime} \gamma^{\prime}\left(\boldsymbol{F}_{b}+\boldsymbol{H}_{1 c}\right)$

$$
\begin{gathered}
=\alpha^{\prime 2} \boldsymbol{l}+\boldsymbol{\beta}^{\prime 2} \boldsymbol{m}+\boldsymbol{\gamma}^{\prime 2} \boldsymbol{n}+\alpha^{\prime} \beta^{\prime}\left(\boldsymbol{H}+\boldsymbol{H}_{\mathbf{1}}\right)+\alpha^{\prime} \boldsymbol{\gamma}^{\prime}\left(\boldsymbol{G}_{1}+\boldsymbol{G}\right)+\boldsymbol{\beta}^{\prime} \gamma^{\prime}\left(\boldsymbol{F}+\boldsymbol{F}_{\mathbf{1}}\right) \\
-\left(\boldsymbol{\alpha}^{\prime} \boldsymbol{A}+\boldsymbol{\beta}^{\prime} \boldsymbol{B}+\boldsymbol{\gamma}^{\prime} \boldsymbol{C}\right)\left(\alpha^{\prime} \boldsymbol{a}+\boldsymbol{\beta}^{\prime} \boldsymbol{b}+\boldsymbol{\gamma}^{\prime} \boldsymbol{c}\right),
\end{gathered}
$$

(15.) $\Sigma x^{\prime} \boldsymbol{Y}^{\prime}=\boldsymbol{H}^{\prime}=\alpha \alpha^{\prime} \boldsymbol{l}_{a}+\beta \beta^{\prime} m_{b}+\gamma \gamma^{\prime} \boldsymbol{n}_{c}$

$$
\begin{aligned}
& +\alpha \beta^{\prime} \boldsymbol{H}_{a}+\alpha^{\prime} \beta \boldsymbol{H}_{1 b}+\alpha \gamma^{\prime} \boldsymbol{G}_{1 a}+\alpha^{\prime} \gamma \boldsymbol{G}_{c}+\beta \gamma^{\prime} \boldsymbol{F}_{b}+\beta^{\prime} \gamma \boldsymbol{F}_{1 c} \\
& =\alpha \alpha^{\prime} \boldsymbol{l}+\beta \beta^{\prime} \boldsymbol{m}+\gamma \gamma^{\prime} \boldsymbol{n} \\
& +\alpha \beta^{\prime} \boldsymbol{H}+\alpha^{\prime} \beta \boldsymbol{H}_{1}+\alpha \gamma^{\prime} \boldsymbol{G}_{1}+\alpha^{\prime} \boldsymbol{G}+\beta \gamma^{\prime} \boldsymbol{F}+\beta^{\prime} \gamma \boldsymbol{F}_{1} \\
& \quad-\left(\alpha^{\prime} \boldsymbol{A}+\beta^{\prime} \boldsymbol{B}+\gamma^{\prime} \boldsymbol{C}\right)(\alpha \boldsymbol{a}+\beta \boldsymbol{b}+\gamma \boldsymbol{c}),
\end{aligned}
$$

(16.) $\Sigma y^{\prime} \boldsymbol{X}^{\prime}=\boldsymbol{H}_{\mathbf{1}}^{\prime}=\alpha \alpha^{\prime} \boldsymbol{l}_{a}+\boldsymbol{\beta} \boldsymbol{\beta}^{\prime} \boldsymbol{m}_{b}+\gamma \gamma^{\prime} \boldsymbol{n}_{c}$

$$
\begin{array}{r}
+\alpha \beta^{\prime} \boldsymbol{H}_{1 b}+\alpha^{\prime} \beta \boldsymbol{H}_{a}+\alpha \gamma^{\prime} \boldsymbol{G}_{c}+\alpha^{\prime} \gamma \boldsymbol{G}_{\mathbf{1}}+\beta \gamma^{\prime} \boldsymbol{F}_{\mathbf{1}}+\beta^{\prime} \gamma \boldsymbol{F}_{b} \\
=\alpha \alpha^{\prime} \boldsymbol{l}+\boldsymbol{\beta} \boldsymbol{\beta}^{\prime} \boldsymbol{n}+\gamma \gamma^{\prime} \boldsymbol{n} \\
+\alpha \beta^{\prime} \boldsymbol{H}_{\mathbf{1}}+\alpha^{\prime} \boldsymbol{\beta} \boldsymbol{H}+\alpha \gamma^{\prime} \boldsymbol{G}+\alpha^{\prime} \gamma \boldsymbol{G}_{\mathbf{1}}+\beta \gamma^{\prime} \boldsymbol{F}_{\mathbf{1}}+\boldsymbol{\beta}^{\prime} \gamma \boldsymbol{F} \\
-(\alpha \boldsymbol{A}+\boldsymbol{\beta} \boldsymbol{B}+\boldsymbol{C})\left(\alpha^{\prime} \boldsymbol{a}+\boldsymbol{\beta}^{\prime} \boldsymbol{b}+\gamma^{\prime} \boldsymbol{c}\right),
\end{array}
$$

u. s. w.

\section{S. 5.}

Verbindungen dieser Gleichungen zwischen den Momenten der parallelen Seitenkräfte erzeugen wieder neue Gleichungen zwischen den Momenten der nicht zerlegten Kräfte um die Coordinatenaxen zweier Coordinatensysteme; so entstehen durch Abzählen der Gleichungen 1, 2 oder 7, 8, der Gleichungen 3, 4 oder 9, 10 und der Gleichungen 5, 6 oder 11, 12 folgende:

$$
\begin{aligned}
& \left\{\begin{array}{l}
\beta^{\prime \prime} \boldsymbol{N}_{a b}-\gamma^{\prime \prime} \boldsymbol{M}_{a c}=-\alpha \boldsymbol{M}^{\prime}+\alpha^{\prime} \boldsymbol{L}^{\prime} \\
\gamma^{\prime \prime} \boldsymbol{L}_{b c}-\alpha^{\prime \prime} \boldsymbol{N}_{a b}=-\boldsymbol{\beta} \boldsymbol{M}^{\prime}+\boldsymbol{\beta}^{\prime} \boldsymbol{L}^{\prime} \\
\alpha^{\prime \prime} \boldsymbol{M}_{a c}-\boldsymbol{\beta}^{\prime \prime} \boldsymbol{L}_{b c}=-\boldsymbol{\gamma} \boldsymbol{M}^{\prime}+\boldsymbol{\gamma}^{\prime} \boldsymbol{L}^{\prime},
\end{array}\right. \\
& \left\{\begin{array}{l}
\boldsymbol{\beta}^{\prime} \boldsymbol{N}_{a b}-\boldsymbol{\gamma}^{\prime} \boldsymbol{M}_{a c}=+\alpha \boldsymbol{N}^{\prime}-\alpha^{\prime \prime} \boldsymbol{L}^{\prime} \\
\gamma^{\prime} \boldsymbol{L}_{b c}-\alpha^{\prime} \boldsymbol{N}_{a b}=+\boldsymbol{\beta} \boldsymbol{N}^{\prime}-\boldsymbol{\beta}^{\prime \prime} \boldsymbol{L}^{\prime} \\
\alpha^{\prime} \boldsymbol{M}_{a c}-\boldsymbol{\beta}^{\prime} \boldsymbol{L}_{b c}=+\boldsymbol{\gamma} \boldsymbol{N}^{\prime}-\boldsymbol{\gamma}^{\prime \prime} \boldsymbol{L}^{\prime},
\end{array}\right.
\end{aligned}
$$




$$
\left\{\begin{array}{l}
\beta \boldsymbol{N}_{a b}-\gamma \boldsymbol{M}_{a c}=-\alpha^{\prime} \boldsymbol{N}^{\prime}+\alpha^{\prime \prime} \boldsymbol{M}^{\prime} \\
\gamma \boldsymbol{L}_{b c}-\alpha \boldsymbol{N}_{a b}=-\beta^{\prime} \boldsymbol{N}^{\prime}+\beta^{\prime \prime} \boldsymbol{M}^{\prime} \\
\alpha \boldsymbol{M}_{a c}-\beta \boldsymbol{L}_{b c}=-\gamma^{\prime} \boldsymbol{N}^{\prime}+\gamma^{\prime \prime} \boldsymbol{M}^{\prime} .
\end{array}\right.
$$

\$. 6 .

Die Gleichungen für Kräfte in einer Ebene ergeben sich aus den obigen, wenn in ihnen gesetzt wird

$$
\boldsymbol{F}=\boldsymbol{F}_{1}=\boldsymbol{G}=\boldsymbol{G}_{1}=\boldsymbol{n}=\mathbf{0} \text { und } \quad \alpha^{\prime \prime}=\beta^{\prime \prime}=\gamma=\gamma^{\prime}=\mathbf{0}
$$

und auch

$$
\boldsymbol{F}^{\prime}=\boldsymbol{F}_{1}^{\prime}=\boldsymbol{G}^{\prime}=\boldsymbol{G}_{1}^{\prime}=\boldsymbol{n}^{\prime}=\mathbf{0}
$$

nämlich

und

(20.) $\quad\left\{\begin{array}{l}\alpha^{\prime} \boldsymbol{l}_{a}+\beta^{\prime} \boldsymbol{H}_{a}=\alpha \boldsymbol{H}^{\prime}+\alpha^{\prime} m^{\prime} \\ \alpha^{\prime} \boldsymbol{H}_{1 b}+\beta^{\prime} m_{b}=\beta \boldsymbol{H}^{\prime}+\beta^{\prime} m^{\prime}\end{array}\right.$

$$
\left\{\begin{array}{l}
\alpha^{\prime} \boldsymbol{l}_{a}+\beta^{\prime} \boldsymbol{H}_{1 b}=\alpha \boldsymbol{H}_{1}^{\prime}+\alpha^{\prime} \boldsymbol{m}^{\prime} \\
\alpha^{\prime} \boldsymbol{H}_{a}+\beta^{\prime} m_{b}=\beta \boldsymbol{H}_{1}^{\prime}+\beta^{\prime} \boldsymbol{m}^{\prime}
\end{array}\right.
$$

$$
\begin{aligned}
& \left\{\begin{array}{l}
\alpha l_{a}+\beta \boldsymbol{H}_{a}=\alpha l^{\prime}+\alpha^{\prime} \boldsymbol{H}_{1}^{\prime} \\
\alpha \boldsymbol{H}_{1 b}+\beta m_{b}=\beta l^{\prime}+\beta^{\prime} \boldsymbol{H}_{1}^{\prime}
\end{array}\right. \\
& \begin{cases}\alpha \boldsymbol{l}_{a}+\beta \boldsymbol{H}_{1 b}=\alpha l^{\prime}+\alpha^{\prime} \boldsymbol{H}^{\prime} \\
\alpha \boldsymbol{H}_{a}+\beta \boldsymbol{m}_{b}=\beta l^{\prime}+\beta^{\prime} \boldsymbol{H}^{\prime}\end{cases}
\end{aligned}
$$

$$
\left\{\begin{array}{l}
\Sigma x^{\prime} \boldsymbol{X}^{\prime}=l^{\prime}=\alpha^{2} l_{a}+\beta^{2} m_{b}+\alpha \beta\left(\boldsymbol{H}_{a}+\boldsymbol{H}_{1 b}\right) \\
\Sigma y^{\prime} \boldsymbol{Y}^{\prime}=\boldsymbol{m}^{\prime}=\alpha^{\prime 2} \boldsymbol{l}_{a}+\beta^{\prime 2} m_{b}+\alpha^{\prime} \beta^{\prime}\left(\boldsymbol{H}_{a}+\boldsymbol{H}_{1 b}\right) \\
\Sigma x^{\prime} \boldsymbol{Y}^{\prime}=\boldsymbol{H}^{\prime}=\alpha \alpha^{\prime} \boldsymbol{l}_{a}+\beta \boldsymbol{\beta}^{\prime} m_{b}+\alpha \beta^{\prime} \boldsymbol{H}_{a}+\alpha^{\prime} \beta \boldsymbol{H}_{1 b} \\
\Sigma y^{\prime} \boldsymbol{X}^{\prime}=\boldsymbol{H}_{1}^{\prime}=\alpha \alpha^{\prime} l_{a}+\beta \boldsymbol{\beta}^{\prime} \boldsymbol{m}_{b}+\alpha \boldsymbol{\beta}^{\prime} \boldsymbol{H}_{1 b}+\alpha^{\prime} \beta \boldsymbol{H}_{a} .
\end{array}\right.
$$

\$. 7.

Die Gleichungen, welche ich im Obigen mitgetheilt habe, finden mannigfaltige Anwendung, besonders bei der Theorie der Mittelpunkte der parallelen Seitenkräfte, die hierauf folgen, und die ganz auf diese Gleichungen sich gründen wird. Aufserdem gibt es Aufgaben, die durch sie nur ihre Lösung erhalten können. Als Beleg hiezu nur folgende drei Fälle:

Die Kräfte eines Kräftensystems nach dreien zu einander senkrechten Richtungen serlegen, so da/s eine von den dreien Gruppen paralleler Seitenkräfle keinen Einflu/s auf das Drehmoment des Kräftensystems hat.

Soll die Gruppe $Z^{\prime}$ keinen Einflufs auf das Drehmoment haben, so

fällt diese Aufgabe mit folgender zusammen: Das Coordinatensystem so Crelle's Journal f. d. M. Bd. XLVII. Heft 3. 
umzuändern, dafs

$$
\Sigma x^{\prime} \boldsymbol{Z}^{\prime}=\Sigma y^{\prime} \boldsymbol{Z}^{\prime}=0 \quad \text { oder } \quad G_{i}^{\prime}=F^{\prime}=0 .
$$

Die Gleichungen N.1 lösen diese Aufgabe, sie sind in diesem Falle

$$
\begin{gathered}
\alpha^{\prime \prime} l+\beta^{\prime \prime} \boldsymbol{H}_{a}+\gamma^{\prime \prime} \boldsymbol{G}_{1 a}=\alpha^{\prime \prime} n^{\prime} \\
\alpha^{\prime \prime} \boldsymbol{H}_{1 b}+\beta^{\prime \prime} m_{b}+\gamma^{\prime \prime} \boldsymbol{F}_{b}=\beta^{\prime \prime} n^{\prime} \\
\alpha^{\prime \prime} \boldsymbol{G}_{c}+\beta^{\prime \prime} \boldsymbol{H}_{1 c}+\gamma^{\prime \prime} \boldsymbol{n}_{c}=\gamma^{\prime \prime} \boldsymbol{n}^{\prime} .
\end{gathered}
$$

$\mathrm{Zu}$ diesen kommt noch die Gleichung

$$
1=\alpha^{\prime \prime 2}+\beta^{\prime \prime 2}+\gamma^{\prime \prime 2} \text {. }
$$

Die Verbindung dieser vier Gleichungen führt zu den Werthen von $\alpha^{\prime \prime}, \beta^{\prime \prime} \gamma^{\prime \prime}$, welche die Coordinaten $a, b, c$ des Anfangspunktes des zweiten Coordinatensystems unbestimmt lassen.

S. 8.

Sollen die Kräfte eines Kräftensystems in drei Gruppen paralleler Seitenkräfte zerlegt werden, so dafs das Drehmoment der Gruppe $\boldsymbol{X}^{\prime}$ um die Axe $y^{\prime}$ und das Drehmoment der Gruppe $\boldsymbol{Y}^{\prime}$ um die Axe $\boldsymbol{x}^{\prime}$ keinen Einflufs auf das Drehmoment des ganzen Kräftensystems hat, so hat man in N. 2

$$
\Sigma \mathfrak{z}^{\prime} \boldsymbol{X}^{\prime}=\Sigma \mathfrak{z}^{\prime} \boldsymbol{Y}^{\prime}=\mathbf{0} \quad \text { oder } \quad \boldsymbol{G}^{\prime}=\boldsymbol{F}_{1}^{\prime \prime}=\mathbf{0}
$$

zu setzen. Da wenigere Gleichungen als zu bestimmende Gröfsen $\alpha^{\prime \prime}, \beta^{\prime \prime}, \gamma^{\prime \prime}$, $a, b, c$ vorhanden, so können mit den gestellten Bedingungen noch andere verbunden werden.

S. 9.

Können die Kräfte eines Kräftensystems in drei Gruppen paralleler Seitenkräfte zerlegt werden, so dafs das Drehmoment zweier Gruppen $\boldsymbol{X}^{\prime}$ und $\boldsymbol{Z}^{\prime}$ um die Axe $y^{\prime}$ und zwar das Drehmoment einer jeden von ihnen $=0$ ist?

Nach diesen Bestimmungen soll nicht allein

$$
\Sigma\left(\boldsymbol{z}^{\prime} \boldsymbol{X}^{\prime}-\boldsymbol{x}^{\prime} \boldsymbol{Z}^{\prime}\right)=0
$$

sondern noch

$$
\Sigma \boldsymbol{z}^{\prime} \boldsymbol{X}^{\prime}=\Sigma \boldsymbol{x}^{\prime} \boldsymbol{Z}^{\prime}=\mathbf{0} \text { oder } \quad \boldsymbol{G}^{\prime}=\boldsymbol{G}_{1}^{\prime}=\mathbf{0} .
$$

Diese Frage wird entschieden durch die Gleichungen 1 und 2 , wenn in ihnen die vorstehenden Werthe gesetzt werden. Durch Elimination von $F^{\prime \prime}$ und $n^{\prime}$ erhält man aus ihnen die beiden Gleichungen

$$
\left.\left.\begin{array}{rr}
\left(\alpha^{\prime \prime} l_{a}+\beta^{\prime \prime} \boldsymbol{H}_{a}+\gamma^{\prime \prime} \boldsymbol{G}_{1 a}\right) \alpha \\
+\left(\alpha^{\prime \prime} \boldsymbol{H}_{1 b}+\beta^{\prime \prime} m_{b}+\gamma^{\prime \prime} \boldsymbol{F}_{b}\right) \boldsymbol{\beta} \\
+\left(\alpha^{\prime \prime} \boldsymbol{G}_{c}+\boldsymbol{\beta}^{\prime \prime} \boldsymbol{F}_{1 c}+\gamma^{\prime \prime} \boldsymbol{n}_{c}\right) \gamma
\end{array}\right\}=0, \quad \begin{array}{r}
\left(\alpha^{\prime \prime} \boldsymbol{l}_{a}+\beta^{\prime \prime} \boldsymbol{H}_{1 b}+\gamma^{\prime \prime} \boldsymbol{G}_{c}\right) \boldsymbol{\alpha} \\
+\left(\alpha^{\prime \prime} \boldsymbol{H}_{a}+\beta^{\prime \prime} \boldsymbol{m}_{b}+\gamma^{\prime \prime} \boldsymbol{F}_{1 c}\right) \boldsymbol{\beta} \\
+\left(\alpha^{\prime \prime} \boldsymbol{G}_{1 a}+\boldsymbol{\beta}^{\prime \prime} \boldsymbol{F}_{b}+\gamma^{\prime \prime} \boldsymbol{n}_{c}\right) \boldsymbol{\gamma}
\end{array}\right\}=0,
$$


welche in Verbindung mit den Gleichungen

$$
1=\alpha^{2}+\beta^{2}+\gamma^{2}, \quad 1=\alpha^{\prime \prime 2}+\beta^{\prime \prime 2}+\gamma^{\prime \prime 2}
$$

vielen Spielraum für die zu bestimmenden Gröfsen lassen.

Durch Abzählen der beiden ersten Gleichungen entsteht die Gleichung

$$
\alpha^{\prime} \boldsymbol{L}_{b c}+\beta^{\prime} \boldsymbol{M}_{a c}+\gamma^{\prime} \boldsymbol{N}_{a b}=0
$$

welche angiebt, dafs die Coordinatenaxe $y^{\prime}$ in der Momentenebene des Drehpunkts $(a b c)$ liegt, und durch diesen Punkt geht.

$\mathrm{Zu}$ demselben Schlusse würde man gekommen sein, wenn man von den Gleichungen $\$$. 5 Anwendung gemacht hätte.

Heidelberg, im December 1852. 